\title{
Professionalism of speech pathologist as a member of multidisciplinary team: competence in dysphagia correction
}

\author{
D.V. Uklonskaya ${ }^{1 *}$, V.E. Agaeva ${ }^{2}$, and Yu.A. Pokrovskaya ${ }^{3}$ \\ ${ }^{1}$ Central Clinical Hospital "RZD-Medicine", Moscow, Russia \\ ${ }^{2}$ Moscow City University, Moscow, Russia \\ ${ }^{3}$ Moscow City University, Moscow, Russia
}

\begin{abstract}
The article is devoted to an important factor in successful career of speech pathologist - professionalism within the work of multidisciplinary team of specialists. On the example of integrated approach in the correction of swallowing disorders, the authors proved the effectiveness of speech therapy technologies in treatment, rehabilitation and social adaptation process. Additions to the structure of the competence of speech pathologist in helping people with dysphagia, presented through the totality of organization and methodological content of correctionalpedagogical classes, ethics and deontology of relations with microsocial circle, have been made. Purpose: assessment of the specifics of meal disorders and improving the organization of pedagogical rehabilitation of patients with paresis and paralysis of larynx of peripheral genesis within interaction of specialists in multidisciplinary team.
\end{abstract}

\section{A problem statement}

Speech pathologist, as a specialist, engaged in the prevention, detection and elimination of speech disorders, implements the main directions of his professional activity (diagnostic, correctional-developmental, organizational-methodological, consultative-educational, preventive) in educational organizations, healthcare organizations and social protection institutions with group representatives of various both by age and by psychophysical status. In particular, modern technologies for the comprehensive rehabilitation of people with changes in somatic, psychosomatic and psychoneurological profiles require the inclusion of speech pathologist in multidisciplinary team, that carries out treatment, rehabilitation and social adaptation process [1-3].

Dysphagia is a swallowing disorder, which can be caused by congenital or acquired diseases of the digestive system, foreign bodies, cicatricial changes after injuries or removals of neoplasms, leading to defects and deformities of the maxillofacial area, congenital cleft lip and cleft palate, some diseases of the central nervous system, iatrogenic nerve disorders, that provide mobility of organs involved in the swallowing act, etc. [4-7].

*Corresponding author: ipcs-profped@yandex.ru 
Since these are anatomical areas, important for chewing, swallowing, breathing and speaking, the inevitable disorders of above functions significantly aggravate inanition of the body and complicate the treatment and rehabilitation process $[6,7]$.

At the same time, it should be emphasized that the meal process is an important component not only of the quality of life, but also of social integration. Overcoming such disorder requires not only medical intervention, but also adaptation measures [8-11]. The inclusion of speech pathologist in recovery activities, that improve the quality of life and socialization of people with swallowing pathology of various etiologies, significantly expands rehabilitation capabilities.

Despite the developed current methods of treatment, there are a number of unresolved issues related to the recovery of the functions of the peripheral division of speech apparatus of people in this category (swallowing, breathing, speech) and to the harmonization of their psycho-emotional background. Further research in the development of directions for speech therapy and in the practical use of methods and techniques in correctional work, necessary for the optimization and effective rehabilitation of people with dysphagia, is required. The results should facilitate expanding and clarifying the professional competence of speech pathologist, increasing his professionalism and being in demand as a member of multidisciplinary team.

Our experience of participating in multidisciplinary team for comprehensive rehabilitation of people with iatrogenic limitation of laryngeal mobility showed that correctional-pedagogical work to eliminate dysphagia should be an integral part of logopaedic activities to normalize speech function in patients of the above group $[12,13]$. Available literature indicates breathing and voice disorders as accompanying acquired disorders of laryngeal mobility, but there are no indications of meal disorders, and there are no practical recommendations for their elimination. Meanwhile, practical experience allows us to affirm that the majority of patients with limited mobility of larynx experience difficulties in swallowing, which do not disappear on their own and require special correction. In this case, we can talk about the phenomenon of two "D" - dysphonia, dysphagia - often combined with breathing disorders, and accompanying acquired paresis and paralysis of larynx of peripheral genesis.

In our article we will consider mechanisms of meal disorders in this category of people and the effectiveness of proposed directions of correctional logopaedic work, aimed at overcoming these disorders, knowledge of which will increase the professionalism of speech pathologist.

Thus, the purpose of our work is to assess the specifics of meal disorders and improve the organization of pedagogical rehabilitation of patients with paresis and paralysis of larynx of peripheral genesis within interaction of specialists in multidisciplinary team.

\subsection{The objective of the work}

From 2017 to 2019, an open, non-randomized, longitudinal, panel, controlled study was conducted at PHF "Central Clinical Hospital "RZD-MEDICINE". Examination of 86 people with voice, breathing and swallowing disorders after thyroid surgery (18 men and 68 women) was conducted. The entry criterion was a disorder of pharyngeal phase of swallowing cycle. Subsequently, 16 people were excluded from the study due to the impossibility to conduct speech rehabilitation because of economic factors or because of need to continue treatment of the basic disease; 2 of them refused without explanation.

The experimental group (I) included patients who underwent a full course of speech therapy to correct peripheral neurogenic dysphagia: 50 people ( 8 men (16\%), 42 women $(84 \%))$. The average age is 54.6 years. 
The control group (II) included 20 people (4 men (20\%), 16 women (80\%)) with similar swallowing disorders who could not receive logopaedic help in full for various reasons: the meal act was examined and counseling was held. The average age is 54.4 years.

The groups were balanced according to the severity of defect, gender and age (Student's t-test-1.68. $\mathrm{p}=0.108048$ ). The repeated block of examination of swallowing act was carried out in both groups during planned hospitalization after 3 months.

The examination and management of the patients were carried out by multidisciplinary team of specialists. In all patients observed within postoperative examination of speech status after endoscopic examination of larynx with an assessment of its functional activity, together with surgeon - the meal process was assessed by testing the process of swallowing food and liquids of various consistencies (Volume Viscosity Swallow Test (V-VST) and by swallowing assessment with the Total Dysphagia Severity Scale [4]. According to this scale: 0 points were regarded as lack of dysphagia; 1 point - mild dysphagia, 2 points moderate dysphagia; 3 points - severe dysphagia; 4 points - very severe dysphagia, inability to meal orally and the need for nasogastric tube or gastrostomy.

When dysphagia was detected within speech therapy, differentiated work by stage and severity, including the use of compensatory, adaptive and recovery strategies, was carried out to eliminate meal disorders [8-10].

For data processing we used the statistical package IBM SPSS Statistics 22.0.

\section{Materials and the results of the research}

In patients with acquired disorders of meal process due to limited mobility of the larynx, difficulties were registered in the pharyngeal stage of swallowing; and the severity of dysphagia was in direct proportion to the position of paralyzed vocal fold and was aggravated in the case of bilateral damage. Thus, it can be affirmed that swallowing disorders were observed in the intraglottic phase. The number of patients with one or another limitation of mobility of the larynx is presented in Table 1. It can be seen that groups I and II are comparable in frequency of occurrence of various disorders.

Table 1. The nature of the severity of motor disorders in paresis / paralysis of the larynx of peripheral genesis.

\begin{tabular}{|l|l|l|l|}
\hline Damaged side & $\begin{array}{l}\text { Group } \\
(\mathbf{n}=\mathbf{5 0})\end{array}$ & $\begin{array}{l}\text { Group II } \\
(\mathbf{n}=\mathbf{2 0})\end{array}$ & $\begin{array}{l}\text { Total sample } \\
(\mathbf{n}=\mathbf{7 0})\end{array}$ \\
\hline $\begin{array}{l}\text { Left-side paresis / paralysis of the larynx } \\
\text { ( } 1 \text { subgroup) }\end{array}$ & $20(40 \%)$ & $8(40 \%)$ & $28(40 \%)$ \\
\hline $\begin{array}{l}\text { Right-side paresis / paralysis of the larynx } \\
\text { (2 subgroup) }\end{array}$ & $22(44 \%)$ & $8(40 \%)$ & $30(42,9 \%)$ \\
\hline $\begin{array}{l}\text { Both-side paresis / paralysis of the larynx } \\
\text { (3 subgroup) }\end{array}$ & $8(16 \%)$ & $4(16 \%)$ & $12(17,1 \%)$ \\
\hline
\end{tabular}

Data on the degree of dysphagia by Total Dysphagia Severity Scale for the sample as a whole $(n=70)$ allow to speak about depending the degree of manifestation of disorder on the location and volume of anatomical defect. The Mann-Whitney U-test for independent samples is significant when comparing subgroups 1 and $3(p<0.0001)$, as well as subgroups 2 and $3(p<0.0001)$, and is not significant when comparing subgroups 1 and $2(p<0.249)$, which suggests that it is the both-side paresis/paralysis that aggravates the severity of dysphagia.

These data were taken into account in strategy development of rehabilitation measures.

Then, a course of speech therapy sessions with the participants of group I was conducted. It was aimed at normalizing the meal process. Speech therapy began at hospital, where classes were carried out daily for 7-10 days, then continued on outpatient basis, on 
average 2-3 times a week for 2 months. At the end of the course to normalize the swallowing act, after 3 months, the meal process was assessed again.

The content of speech therapy for the correction of peripheral neurogenic dysphagia is correctional-pedagogical work in the following directions:

- Diet method: in order to alleviate the patient's physical and psychological discomfort at the first stages of comprehensive rehabilitation, the speech pathologist's tasks include determining the optimal consistency of food and recommendations for the patient and his inner circle about the organization of meal process.

- Psychological support: the participation of psychologist is necessary within comprehensive rehabilitation in order to form informational, motivational and methodological competence in patient and his microsocial circle.

- Physiotherapy methods (after discussion with the surgeon).

- Active / passive static and dynamic gymnastics of the articulation organs, face, neck and shoulder girdle, breathing exercises.

-Logopaedic correction aimed at recovery of pharyngeal reflex and normalizing the swallowing act; recovery of pharyngeal activity, normalization of speech, physiological breathing, functional activity of the muscles involved in swallowing act and coordination of breathing, swallowing and speech processes.

The main purpose of pedagogical impact was the activation of pharyngeal muscles and recovery of movement coordination of pharynx and larynx. Correction of acquired disorders was carried out on the basis of long-term experience of normal functioning of the muscles, involved in swallowing act, that made it possible to build recovery training, taking into account the previously fixed, firmly established skills.

Vigorous activity in postoperative period allows the patient to escape from heavy thoughts, and achieved improvements instill self-confidence, hope for success, thereby changing the patient's emotional sphere. Nevertheless, throughout full speech therapy course, we consider it necessary to conduct conversations aimed at providing emotional support and the formation of desire for soonest rehabilitation of lost functions. The main leitmotif of such conversations is the setting to shorten the rehabilitation time, to fastest possible inclusion to active life, to returning to usual duties or getting closer to them.

It is known that somatically weakened organism has reduced opportunities for spontaneous compensation, and this leads to the formation of pathological stereotypes. Despite the fact that early speech therapy is associated with some difficulties due to the healing process of postoperative wound, it is necessary to start them as early as possible in order to prevent the formation of pathological habits and to help the patient to adapt to his new condition, to nutrition through natural ways and to achieve fast recovery of lost skills.

One of the important principles which the work with this contingent is based on, is the load dosage. Since the patient's asthenization after surgery get worse even more as the degree of dysphagia increases, we conducted speech therapy in sparing mode, giving the fractional load, gradually increasing the training time, at the same time complicating the material and implementing the succession of recovery stages. So, 2-3 short sessions of 3-7 minutes were conducted per day, with breaks of at least 30 minutes.

An important feature of working with this category of patients is the forced refusal of active stimulation in the neck muscles, especially with the help of various kinds of hardware techniques, since in most cases in the group of patients there are people with "malignant tumor" diagnosis.

Taking into account the complexity of defect, somatic weakness and psychological characteristics of patients, we worked with each of them individually, implementing a strictly differentiated approach when correcting disorders in each case. It should be noted that the patient's first successes and visible evidence of the effectiveness stimulate further desire to work and significantly improve the psychological condition. 
When eliminating neurogenic disorders in meal process of peripheral genesis by methods of pedagogical impact, we observed the following stages:

1. Preparatory:

- preoperative (establishing contact, assessing speech status, training physiologically conditioned phonation breathing, conversations aimed at developing motivation for rehabilitation);

- postoperative (questioning, logopaedic examination, further training of physiologically conditioned phonation breathing and differentiation of oral and nasal exhalation, nutritional recommendations (food consistency) and conversations aimed at supporting and developing motivation for rehabilitation).

2. The stage of active training (recovery of the functional activity of muscles involved in the swallowing act with the help of static and dynamic articulatory gymnastics, conversations aimed at further improving motivation for rehabilitation).

3. Consolidation of the recovered skills and the formation of stable stereotype of "safe" swallowing (coordination of swallowing, phonation and breathing), vocal exercises using first row vowels separately and in combinations (for severe vocal disorders, silent articulation was offered), conversations aimed at improving motivation for rehabilitation.

Since specialists in psychological and pedagogical support often have to contact the patient's family and the inner circle, we assumed that tactically correct behavior of doctors and speech pathologists should have positive effect on the process of rehabilitation and resocialization on the whole. Our practical experience has shown that the microsocial circle has significant impact on the success of resocialization. This speaks of the need to develop correctional-pedagogical direction, including technologies for involving family members in the rehabilitation and recovery process [14-16].

In the correctional-pedagogical work on eliminating neurogenic peripheral dysphagia, we used the following technologies to involve family members in the rehabilitation and recovery process.

1. Informing family members about the causes and mechanisms of disorders that have arisen, the specifics of the disease, the stages and content of the rehabilitation process.

2. Motivation for conscious active involvement in the recovery process, the formation of correct attitude towards patient, the perception of him as a full-fledged person who temporarily needs additional care, but has the opportunity to return to his usual way of life.

3. Training in elementary corrective techniques, which consist in recommendations for care (diet specifics, feeding methods, oral care, etc.), articulation exercises, breathing and vocal gymnastics.

The following organizational forms of work with family members of patient, who undergoing rehabilitation program, are recommended.

1. Individual: conversations that form the informational and motivational components of the competence of microsocial circle; consulting and training sessions, that introduce relatives to elementary correctional techniques; the presence of relatives in speech therapy classes; sharing homework.

2. Collective: open classes; conferences; meetings; clubs; organization of leisure activities; public organizations and foundations. This form of work allows to normalize intra-family relations, reduces negative psychological background in family and contributes to patient's resocialization.

3. Visual: information stands and table information; Internet resources; telemedicine technologies; online-counseling. The use of these forms allows to expand information competence of all members of microsocial circle and makes communication with qualified specialist and people in a similar life situation more accessible.

At the end of the course to normalize swallowing act, the meal process was assessed again (Figure 1). 


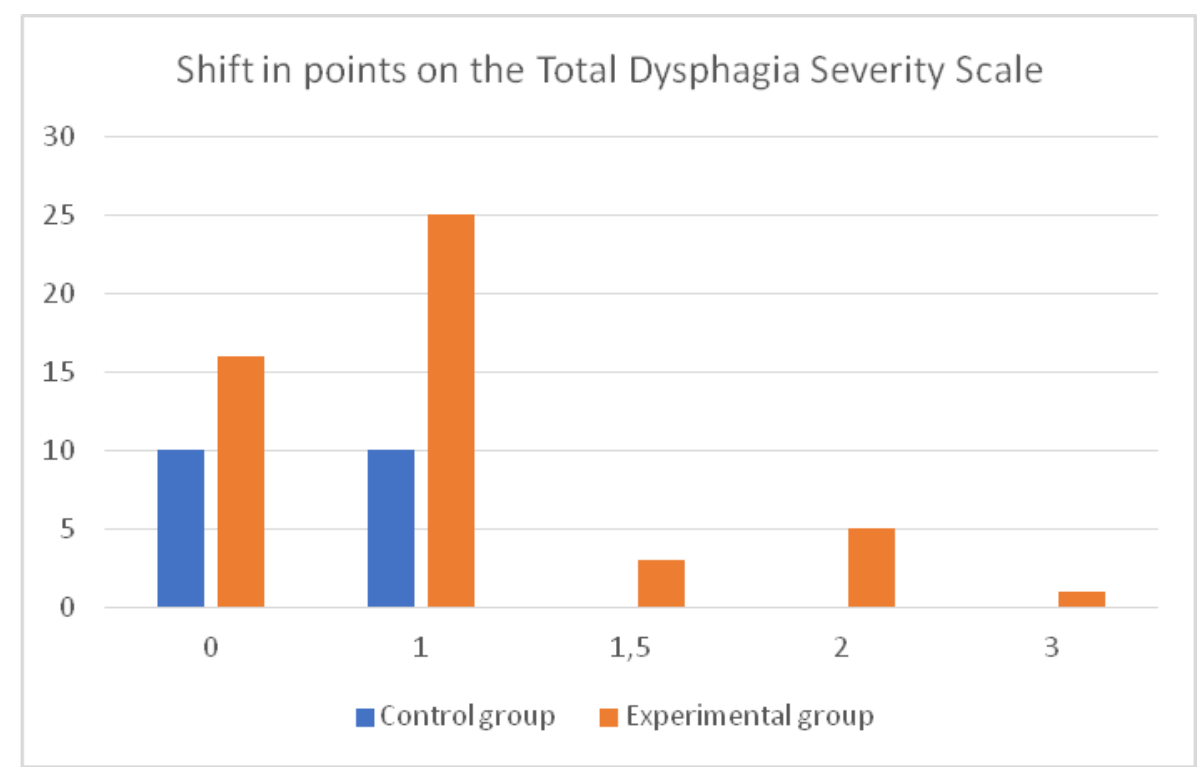

Fig. 1. The results of comparing data of re-assessment of the dysphagia severity in two groups of patients (I and II).

The results of comparing data of re-assessment of the dysphagia severity in two groups of patients (I and II) showed that rehabilitation measures carried out in group I had a positive effect. Differences in overall scale shift for assessing the severity of dysphagia [1] (the difference in points between the first and second assessments of dysphagia degree) between the groups were significant (The Mann-Whitney U-test, $p<0.0001$ ).

Based on the above, we consider it appropriate to present the structure of professional competence of speech pathologist in helping people with swallowing disorders, through the totality of organization and methodological content of correctional-pedagogical classes, ethics and deontology of relations with microsocial circle. It should consist of the following components.

Educational competencies (EC):

- the ability to analyze, systematize and use modern knowledge about the causes and mechanisms of swallowing disorders, about ways to include the microsocial circle in the rehabilitation process and its role in socialization process; domestic and foreign experience in organizing comprehensive help to people of this category;

- the ability to carry out social interaction with people with swallowing disorders and their inner circle;

- the ability to fulfill their role in multidisciplinary team.

General professional competencies (GPC):

- the ability to carry out professional activities in accordance with regulatory legal acts and standards of professional ethics;

- the ability to control and evaluate the effectiveness of work, modify existing methodical techniques depending on individual capabilities and needs of the subject of correction;

- the ability to use modern technologies necessary for individualization of rehabilitation measures;

- the ability to interact with participants in comprehensive rehabilitation process as part of the implementation of the speech therapy support program;

- the ability to carry out professional activities based on special scientific knowledge.

Professional competencies (PC): 
- the ability to carry out speech therapy for recovery and rehabilitation of people with swallowing disorders in healthcare organizations, taking into account the nosological group;

- the ability to carry out psychological-pedagogical support and prevention of secondary (psychoemotional) disorders in people with dysphagia, experiencing difficulties in social adaptation.

\section{Conclusions}

In patients with acquired swallowing disorders of peripheral genesis, there are "intraglottic" disorders of meal act, requiring immediate measures to normalize. Moreover, bilateral damage of larynx aggravates the severity of dysphagia. Since the meal process is a vital function, overcoming nutritional disorders in postoperative period is an integral part of speech therapy of acquired swallowing disorders. Logopaedic impact, which refers to nondrug, non-invasive methods of rehabilitation, includes the use of compensatory, adaptive and recovery strategies. This makes it possible to normalize the meal process successfully, helps to prevent cachexia-anorexia and dehydration, that is important for successful postoperative period, as well as improving the quality of life of patients.

To improve professional competence, speech pathologist must have knowledge, skills and abilities that allow to normalize swallowing disorders successfully using correctionalpedagogical methods. The activity of speech pathologist in healthcare organizations fully corresponds to his functional responsibilities, and competence in the use of knowledge in multidisciplinary disciplines, increasing the effectiveness of rehabilitation measures, contributes to the growth of his professionalism, demand and objectification of inclusion in multidisciplinary team.

\section{References}

1. I.A. Avdyunina, Swallowing disorders in diseases of the nervous system. In the book Rehabilitation of neurological patients tients, p. 393-445 (Medpress-inform, 2008).

2. O.S. Orlova, I.Yu. Levchenko, I.V. Evtushenko, Questions of the content of the professional standard "teacher-defectologist", Modern problems of science and education, 6, 405 (2015)

3. E.V. Koryakova, The importance of integrating psychological and pedagogical disciplines in the effective work of a speech therapist, Modern trends and innovations in the humanity and social sciences, IV, 291-297 (2019)

4. J.A. Logemann, Evaluation and treatment of swallowing disorders, p. 406 (Austin, Tex: PRO-ED, 1998)

5. V. Guntram, Diagnosis and treatment of neurogenic dysphagia, p. 77-88 (BremenLondon-Boston: UNI-MED, 2011)

6. V.E. Agaeva, T.Ya. Mos'pan, Speech therapy behavior correction in children with congenital disorders of the lips and palate, Problems of modern pedagogical education, 63-2, 10-13 (2019)

7. E. Bruno, Practical guide to the diagnosis and rehabilitation of impaired swallowing (based on research and practice in the United States), p. 61 (Moscow, 2015).

8. K. Lyozer, Y.A. Adends, Y.U. Hyubner, Nutrition in nowadays oncology, p. 128 (Bremen: UNI-MED, 2013) 
9. N.A. Osipova, I.V. Reshetov, V.V. Sokolov, M.A. Pankratova, M.M. Filyushin, T.V. Dolgopolova, F.E. Sevryukov, Enteral nutrition support in the surgery of head and neck tumors, Oncological Surgery, 2 (4), 5-22 (2010)

10. M. Elia, Screening for malnutrition: A multidisciplinary responsibility. Development and Use of the Malnutrition Universal Screening Tool ('MUST') for Adults, p. 5 (BAPEN, United Kingdom, 2003).

11. G. Nitenberg, B. Raynard, Nutritional support of the cancer patient: issues and dilemmas, Crit. Rev. Oncol. Hematol, 34, 137 (2000)

12. D.V. Uklonskaya, V.E. Agaeva, Normalization of swallowing as a way to optimize speech therapy behavior in maxillofacial defects, Problems of modern pedagogical education, 55-10, 190-196 (2017)

13. D.V. Uklonskaya, Y.A. Pokrovskaya, V.E. Agaeva, The main directions of speech therapy for the correction of neurogenic dysphagia in paresis and paralysis of the larynx of peripheral Genesis, Special education, 3 (55), 92-104 (2019)

14. D.V. Uklonskaya, V.E. Agaeva, To the question of the effectiveness of speech therapy for faces after surgical treatment of the head and neck, Special education, 1 (57), 8495 (2020)

15. O.V. Koshcheeva, Pedagogical ethics as an important component of the professionalism of a speech therapist teacher, Strahov's Readings, 27, 190-197 (2019)

16. T.V. Rogacheva, Rehabilitation potential as a basis for the rehabilitation rout of a disabled person, p. 414-422 (Moscow, 2017) 